\title{
STOCHASTIC COMPARISONS OF NONHOMOGENEOUS PROCESSES
}

Félix Belzunce, Rosa E. Lillo José M. Ruiz and Moshe Shaked

00-13

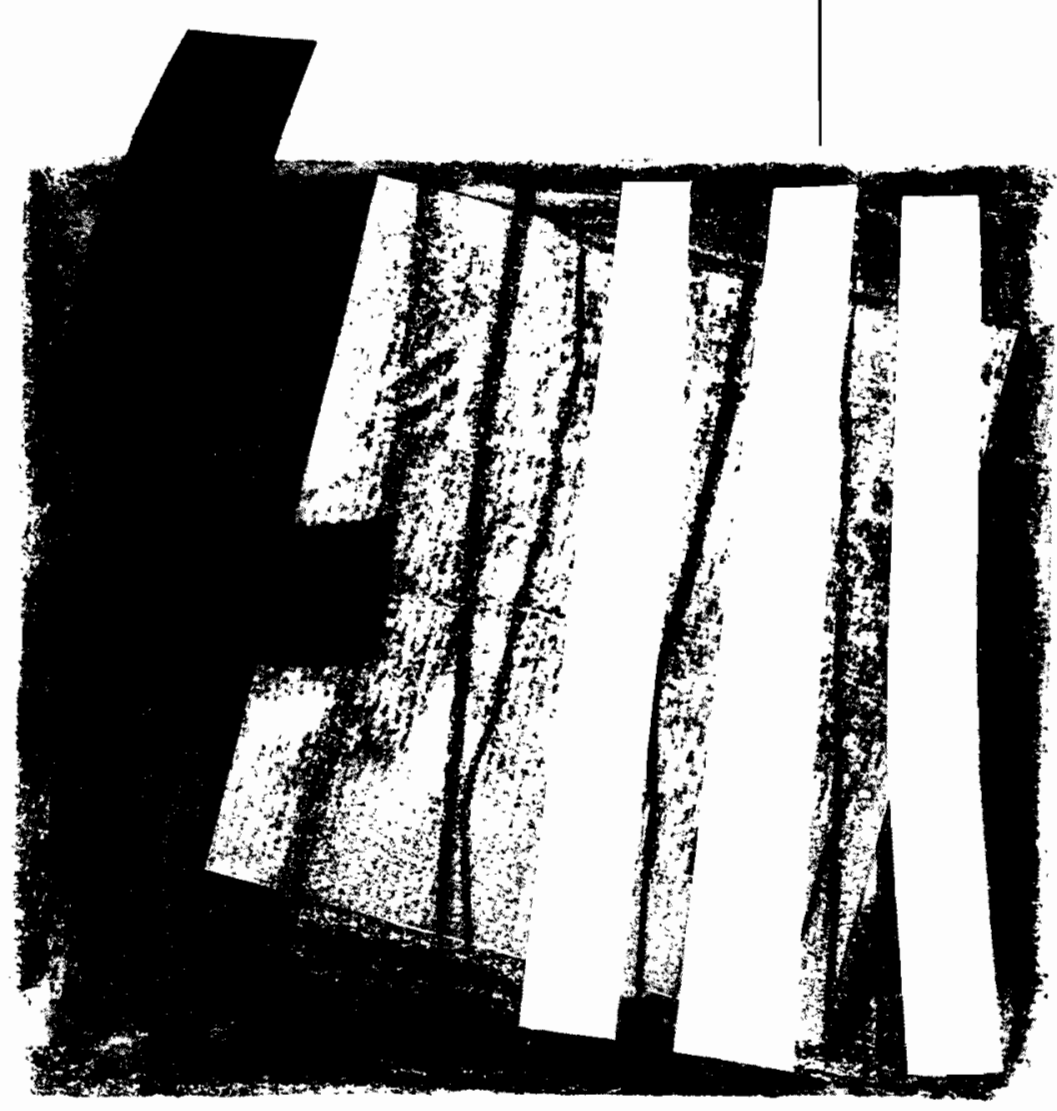




Working Paper 00-13

Statistics and Econometrics Series (7)

February 2000
Departamento de Estadística y Econometría

Universidad Carlos III de Madrid

Calle Madrid, 126

28903 Getafe (Spain)

Fax (34-91) 624-9849

\title{
STOCHASTIC COMPARISIONS OF NONHOMOGENEOUS PROCESSES.
}

Felix Belzunce, Rosa E. Lillo, José M. Ruiz and Moshe Shaked*

\begin{abstract}
The purpose of this paper is to describe various conditions on the parameters of pairs of nonhomogeneous Poisson or birth processes under which the corresponding epoch or inter-epoch times are stochastically ordered in various senses. We derive results involving the usual stochastic order, the multivariate hazard rate order, the multivariate likelihood ratio order, and the multivariate mean residual life order. A sample of applications involving generalized Yule processes, load-sharing models, and minimal repairs in reliability theory, illustrate the usefulness of the new results.
\end{abstract}

Keywords: Nonhomogeneous Poisson process; nonhomogeneous birth process; nonstationary birth process; stochastic order; hazar rate order; likelihood ratio order; mean residual life order; Yule process; load-sharing; minimal repair; dispersive order.

*Belzunce, Dpto. de Estadística e Investigación Operativa, Universidad de Murcia, 30100 Espinardo (Murcia), Spain, e-mail: belzunce@fcu.um.es; Lillo, Departamento de Estadística y.Econometría, Universidad Carlos III de Madrid. C/ Madrid, 126 28903 Madrid. Spain. Ph: 34-91-624.98.57, Fax: 34-91-624.98.49, e-mail: lillo@estecon.uc3m.es; Ruiz, Dpto. de Estadística e Investigación Operativa, Universidad de Murcia, e-mail: jmruizgo@fcu.um.es; Shaked, Department of Mathematics, University of Arizona, Tucson, Arizona 85721, USA, e-mail: shaked@math.arizona.edu. 


\section{Introduction and Motivation}

The nonhomogeneous Poisson process arises naturally in many applications of probability. For example, the epoch times of a nonhomogeneous Poisson process are the times of repair of an item which is being continuously minimally repaired. Also, the epoch times of a nonhomogeneous Poisson process are the consecutive record values of a sequence of independent and identically distributed nonnegative random variables. Therefore, results which give stochastic comparisons of the epoch times or of the inter-epoch times of different nonhomogeneous Poisson processes can be useful in reliability theory and in the studies of progressive records. Other applications of nonhomogeneous Poisson processes are described later in Section 5.

Roughly speaking, the intensity of a jump of a nonhomogeneous Poisson process at any time $t$ depends only on $t$, and not on any other information about the past or the present of the process. A relatively simple extension of this idea gives rise to a large number of other useful processes. These processes will be called below nonhomogeneous birth processes. In a nonhomogeneous birth process the intensity of a jump at any time $t$ depends only on $t$ and on the state (that is, the number of previous jumps) of the process, but not on any other information about the past or the present of the process.

The purpose of this paper is to describe various conditions on the parameters of pairs of nonhomogeneous Poisson or birth processes under which the corresponding epoch or interepoch times are stochastically ordered in various senses.

This paper may be contrasted with the paper of Shaked and Szekli [20, (1995)]. The work [20] focused on the usual stochastic ordering of epoch times and inter-epoch intervals of two point processes, whereas here we derive many results that give finer comparisons in other stochastic ordering senses. However, whereas the results of [20] apply to general point processes, here we derive results only for nonhomogeneous Poisson and birth processes. The results in [20] were mainly applied to comparisons of replacement policies in reliability theory; here we indicate also some other areas of applications of the new results. In fact, we show that the new results here provide useful bounds in almost any area where birth processes are used. Finally, the methods of proof of the present paper differ from the methods of [20]: whereas in [20] most of the basic results were essentially proven using coupling, here we use more analytical methods since most of the stochastic orderings that we derive are stronger than the usual stochastic order, and the tool of coupling does not suffice for their derivation.

In this paper "increasing" and "decreasing" mean "nondecreasing" and "nonincreasing," respectively. For any distribution function $F$ we denote by $\bar{F} \equiv 1-F$ the corresponding 
survival function. Any inverse function that we use below is understood to be the right continuous one.

\section{Nonhomogeneous Poisson and Birth Processes}

A nonhomogeneous Poisson process is parameterized by its intensity (or rate) function $r$. We assume that

$$
\int_{0}^{\infty} r(t) d t=\infty
$$

this ensures that with probability 1 the process has a jump after any time point $t$. A nonnegative function $r$ which satisfies (2.1) can be interpreted as the hazard rate function of a lifetime of an item. More explicitly, if $r$ satisfies (2.1) and we define $f$ by

$$
f(t)=r(t) e^{-\int_{0}^{t} r(u) d u}=r(t) e^{-R(t)}, \quad t \geq 0
$$

where $R(t) \equiv \int_{0}^{t} r(u) d u$, then $f$ is a probability density function of a lifetime; in fact, $f$ is the probability density function of the time of the first epoch of the underlying nonhomogeneous Poisson process. For convenience, if $r\left(t_{0}\right)=\infty$ for some $t_{0}$ then we define $r(t)=\infty$ for $t \geq t_{0}$.

Let $0 \equiv T_{0} \leq T_{1} \leq T_{2} \leq \cdots$ be the epoch times of the nonhomogeneous Poisson process. Denote by $f_{n}$ the density function of $T_{n}, n \geq 1$. Then

$$
f_{n}(t)=f(t) \frac{(R(t))^{n-1}}{(n-1) !}, \quad t \geq 0, \quad n \geq 1
$$

this is (3) in Baxter (1982). Note, in particular, that $f_{1} \equiv f$. It is worthwhile to mention that in the monograph by Kamps (1995) the definition of the epoch times is extended to the so called generalized order statistics; various extensions of (2.3) can be found there.

Let $X_{n}=T_{n}-T_{n-1}, n \geq 1$, be the inter-epoch intervals of the nonhomogeneous Poisson process. Denote by $g_{n}$ the density function of $X_{n}, n \geq 1$. Then $g_{1}=f$ and

$$
g_{n}(t)=\int_{0}^{\infty} r(s) \frac{R^{n-2}(s)}{(n-2) !} f(s+t) d s, \quad t \geq 0, n \geq 2
$$

this is (7) in Baxter (1982).

The nonhomogeneous Poisson process can be generalized to what can be called a nonhomogeneous birth process. Let $\left\{Y_{n}, n=1,2, \ldots\right\}$ be a sequence of independent absolutely 
continuous nonnegative random variables with density, distribution, and hazard rate functions $k_{n}, K_{n}$, and $r_{n}$, respectively. Define

$$
\begin{aligned}
& T_{1}={ }_{\text {st }} Y_{1}, \\
& T_{n}=_{\text {st }}\left[Y_{n} \mid Y_{n}>T_{n-1}\right], \quad n \geq 2,
\end{aligned}
$$

where $=_{\text {st }}$ denotes equality in law, and for any event $A$ the notation $\left[Y_{n} \mid A\right]$ stands for any random variable whose distribution is the conditional distribution of $Y_{n}$ given $A$. The corresponding nonhomogeneous birth process is the process $\{N(t), t \geq 0\}$, with the $T_{n}$ 's as epoch times, defined by

$$
N(t)=\sup \left\{n: T_{n} \leq t\right\}, \quad t \geq 0 .
$$

A nonhomogeneous birth process is parameterized by a sequence $\left\{K_{n}, n=1,2, \ldots\right\}$ of life distributions, or, equivalently, by a sequence $\left\{r_{n}, n=1,2, \ldots\right\}$ of hazard rate functions. Note that the jump intensity of a nonhomogeneous birth process at time $t$ is $r_{n}(t)$ where $n-1$ is the number of epochs before time $t$. Nonhomogeneous birth processes are called 'relevation counting processes' in Pellerey, Shaked and Zinn (1999). When all the $r_{n}$ 's are identical, a nonhomogeneous birth process reduces to a nonhomogeneous Poisson process.

Let $f_{n}$ denote the probability density function of $T_{n}$ defined in (2.5) and (2.6). Then

$$
\begin{aligned}
& f_{1}(t)=k_{1}(t), \quad t \geq 0 \\
& f_{n}(t)=k_{n}(t) \int_{0}^{t} \frac{f_{n-1}(u)}{\bar{K}_{n}(u)} d u, \quad t \geq 0, \quad n \geq 2 .
\end{aligned}
$$

Now let $X_{n}=T_{n}-T_{n-1}, n \geq 1$, be the inter-epoch intervals of the nonhomogeneous birth process, that is,

$$
\begin{aligned}
& X_{1}={ }_{\text {st }} Y_{1}, \\
& X_{n}==_{\text {st }}\left[Y_{n}-T_{n-1} \mid Y_{n}>T_{n-1}\right], \quad n \geq 2 .
\end{aligned}
$$

Then the probability density function $g_{n}$ of $X_{n}$ is given by

$$
\begin{aligned}
& g_{1}(t)=k_{1}(t), \quad t \geq 0 \\
& g_{n}(t)=\int_{0}^{t} k_{n}(u+t) \frac{f_{n-1}(u)}{\bar{K}_{n}(u)} d u, \quad t \geq 0, \quad n \geq 2,
\end{aligned}
$$

where the $f_{n}$ 's are defined in (2.7) and (2.8). 


\section{Stochastic Comparisons of Epoch Times}

\subsection{Nonhomogeneous Poisson processes}

Consider two nonhomogeneous Poisson processes with intensity functions $r$ and $s$, respectively, and with associated density functions (see (2.2)) $f$ and $g$, respectively, and associated distribution functions $F$ and $G$, respectively, and associated cumulative hazard functions $R$ and $S$, respectively. Let the epoch times of the first nonhomogeneous Poisson process be denoted by $T_{1,1} \leq T_{1,2} \leq \cdots$, and let the epoch times of the other nonhomogeneous Poisson process be denoted by $T_{2,1} \leq T_{2,2} \leq \cdots$.

In this subsection we derive some results which stochastically compare vectors of $T_{1, j}$ 's with vectors of $T_{2, j}$ 's.

The first result gives conditions under which the epoch times of the first process are smaller than the epoch times of the other process in the usual stochastic order sense. Recall that a random variable or vector $\boldsymbol{X}$ is smaller in the usual stochastic order than the random variable or vector $\boldsymbol{Y}$ (of the same dimension) if

$$
E \phi(\boldsymbol{X}) \leq E \phi(\boldsymbol{Y})
$$

for all increasing functions $\phi$ for which the above expectations exist. This relationship is usually denoted by $\boldsymbol{X} \leq_{\text {st }} \boldsymbol{Y}$. If the distribution functions of $\boldsymbol{X}$ and $\boldsymbol{Y}$ are $F_{\boldsymbol{X}}$ and $F_{\boldsymbol{Y}}$, respectively, then this relation will sometimes be denoted below by $F_{\boldsymbol{X}} \leq_{\mathbf{s t}} F_{\boldsymbol{Y}}$. The following result is essentially not new.

Theorem 3.1. Let $F$ and $G$ be distribution functions associated with two nonhomogeneous Poisson processes as described above. Then $F \leq_{\mathrm{st}} G$ if, and only if,

$$
-\left(T_{1,1}, T_{1,2}, \ldots, T_{1, n}\right) \leq_{\mathrm{st}}\left(T_{2,1}, T_{2,2}, \ldots, T_{2, n}\right), \quad n \geq 1 .
$$

Note that $F \leq_{\text {st }} G$ in Theorem 3.1 is equivalent to $R \geq S$. Thus Theorem 3.1 is essentially the same as Proposition 3.9 in Shaked and Szekli (1995). Roughly speaking, inequality (3.1) for $n=\infty$ is denoted in Shaked and Szekli (1995) as $N_{1} \geq_{\text {st-D }} N_{2}$, where $N_{1}$ and $N_{2}$ are the underlying nonhomogeneous Poisson processes.

The next result gives conditions under which the epoch times of the first process are smaller than the epoch times of the other process in the multivariate hazard rate order. Shaked and Shanthikumar have given a few equivalent definitions of this order in different papers; see references in page 148 of Shaked and Shanthikumar $[19,(1994)]$. For the purpose 
of this paper we will use the definition given in (4.D.1) of [19]. There is a mistake there. The equation there should be

$$
\eta_{i \mid I \cup J}\left(u \mid \boldsymbol{s}_{I}, \boldsymbol{s}_{J}\right) \geq \lambda_{i \mid I}\left(u \mid \boldsymbol{t}_{I}\right) \quad \text { whenever } I \cap J=\emptyset, \mathbf{0} \leq \boldsymbol{s}_{I} \leq \boldsymbol{t}_{I} \leq u \boldsymbol{e}, \text { and } \mathbf{0} \leq \boldsymbol{s}_{J} \leq u \boldsymbol{e}
$$

where $i \in \overline{I \cup J}$, and $\left(\boldsymbol{s}_{I}, \boldsymbol{s}_{J}\right)$ and $\boldsymbol{t}_{I}$ are possible realizations of the underlying random vectors. Here $\boldsymbol{s}_{I}$ denotes a vector of dimension $|I|$, and similarly $\boldsymbol{s}_{J}$ and $\boldsymbol{t}_{I}$ are defined; $\boldsymbol{e}$ denotes a vector of 1 's of a proper dimension. The functions $\eta_{.}(\cdot \mid \cdot)$ and $\lambda_{.}(\cdot \cdot \cdot)$ are the multivariate conditional hazard rate functions of the two random vectors that are compared; these are defined in (4.C.2) of [19]. When the multivariate conditional hazard rate functions of two random vectors $\boldsymbol{X}$ and $\boldsymbol{Y}$ satisfy (3.2) we will denote this by $\boldsymbol{X} \leq_{\mathrm{hr}} \boldsymbol{Y}$. If the distribution functions of $\boldsymbol{X}$ and $\boldsymbol{Y}$ are $F_{\boldsymbol{X}}$ and $F_{\boldsymbol{Y}}$, respectively, then this relation will sometimes be denoted below by $F_{\boldsymbol{X}} \leq_{\mathrm{hr}} F_{\boldsymbol{Y}}$. In the univariate case the relation $F_{X} \leq_{\mathrm{hr}} F_{Y}$ is equivalent to the requirement that $\bar{F}_{Y} / \bar{F}_{X}$ is an increasing function, and if the corresponding hazard rate functions $r_{X}$ and $r_{Y}$ exist then the relation $F_{X} \leq_{\mathrm{hr}} F_{Y}$ is equivalent to the requirement that $r_{X} \geq r_{Y}$. It is known (see Theorems 4.C.1 and 4.D.1 in [19]) that $\boldsymbol{X} \leq_{\mathrm{hr}}$ $\boldsymbol{Y} \Longrightarrow \boldsymbol{X} \leq_{\text {st }} \boldsymbol{Y}$. Thus, the next result gives a stronger conclusion than Theorem 3.1, but under a stronger assumption.

Theorem 3.2. Let $F$ and $G$ be distribution functions associated with two nonhomogeneous Poisson processes as described above. Then $F \leq_{\mathrm{hr}} G$ if, and only if,

$$
\left(T_{1,1}, T_{1,2}, \ldots, T_{1, n}\right) \leq_{\mathrm{hr}}\left(T_{2,1}, T_{2,2}, \ldots, T_{2, n}\right), \quad n \geq 1
$$

Proof. Fix an $n \geq 1$. Let $\eta \cdot . \cdot(\cdot \mid \cdot)$ be the multivariate conditional hazard rate functions

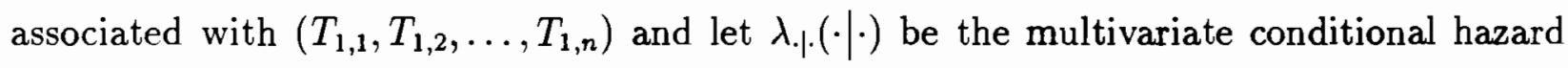
rate functions associated with $\left(T_{2,1}, T_{2,2}, \ldots, T_{2, n}\right)$.

First let us obtain an explicit expression for the right hand side of (3.2). Since $T_{2,1} \leq$ $T_{2,2} \leq \cdots \leq T_{2, n}$ a.s., it follows that $\boldsymbol{t}_{I}$ in (3.2) can be a realization ('history') of observations up to time $u$ only if $I$ is of the form $I=\{1,2, \ldots, m\}$ for some $m \geq 1$, or $I=\emptyset$ (that is, $m=0)$. Then we have

$$
\lambda_{i \mid I}\left(u \mid \boldsymbol{t}_{l}\right)=\left\{\begin{array}{ll}
s(u), & \text { if } i=m+1 ; \\
0, & \text { if } i>m+1 ;
\end{array} \quad \text { where } I=\{1,2, \ldots, m\}\right.
$$

here $s$ is the hazard rate function associated with $G$. 
Next, let us obtain an explicit expression for the left hand side of (3.2). Since $T_{1,1} \leq$ $T_{1,2} \leq \cdots \leq T_{1, n}$ a.s., we see that when $I=\{1,2, \ldots, m\}$ then $\left(\boldsymbol{s}_{I}, \boldsymbol{s}_{J}\right)$ in $(3.2)$ can be a realization of observations up to time $u$ only if $J$ is of the form $J=\{m+1, m+2, \ldots, k\}$ for some $k \geq m+1$, or $J=\emptyset$ (that is, $k=m$ ). Then we have

$$
\begin{aligned}
\eta_{i \mid I \cup J}\left(u \mid \boldsymbol{s}_{I}, \boldsymbol{s}_{J}\right)= \begin{cases}r(u), & \text { if } i=k+1 ; \\
0, & \text { if } i>k+1 ;\end{cases} & \text { where } I=\{1,2, \ldots, m\} \text { and } J=\{m+1, m+2, \ldots, k\} ;
\end{aligned}
$$

here $r$ is the hazard rate function associated with $F$.

Suppose that $F \leq_{\mathrm{hr}} G$. Since $i$ in (3.2) must satisfy $i \in \overline{I \cup J}$ (that is, $i>k$ ), we see that if $k>m$ then

$$
\begin{aligned}
& \eta_{i \mid I \cup J}\left(u \mid \boldsymbol{s}_{I}, \boldsymbol{s}_{J}\right)=r(u) \geq 0=\lambda_{i \mid I}\left(u \mid \boldsymbol{t}_{I}\right) \quad \text { if } i=k+1 \\
& \eta_{i \mid I \cup J}\left(u \mid \boldsymbol{s}_{I}, \boldsymbol{s}_{J}\right)=0=\lambda_{i \mid I}\left(u \mid \boldsymbol{t}_{I}\right) \quad \text { if } i>k+1
\end{aligned}
$$

so (3.2) holds. If $k=m$ (that is, $J=\emptyset$ ) then, using $F \leq_{\mathrm{hr}} G$, we get

$$
\begin{aligned}
& \eta_{i \mid I \cup J}\left(u \mid \boldsymbol{s}_{I}, \boldsymbol{s}_{J}\right)=r(u) \geq s(u)=\lambda_{i \mid I}\left(u \mid \boldsymbol{t}_{I}\right) \quad \text { if } i=k+1 \\
& \eta_{i \mid I \cup J}\left(u \mid \boldsymbol{s}_{I}, \boldsymbol{s}_{J}\right)=0=\lambda_{i \mid I}\left(u \mid \boldsymbol{t}_{I}\right) \quad \text { if } i>k+1
\end{aligned}
$$

so (3.2) holds in this case too.

The necessity part follows from (3.2) with $i=1($ then $I=J=\emptyset)$.

The order $\leq_{h r}$ is not closed under marginalization (though it is closed under the dynamic conditional marginalization described in Shaked and Shanthikumar (1993)). Thus it does not follow from Theorem 3.2 that $T_{1, n} \leq_{h r} T_{2, n}$ for $n \geq 2$ under the conditions stated there. However, in the following result it is shown that this is indeed the case.

Theorem 3.3. Let $F$ and $G$ be distribution functions associated with two nonhomogeneous Poisson processes as described above. Then $F \leq_{\mathrm{hr}} G$ if, and only if, $T_{1, n} \leq_{\mathrm{hr}} T_{2, n}$ for all $n \geq 1$.

Proof. The survival function $\bar{F}_{1, n}$ of $T_{1, n}$ is given by

$$
\bar{F}_{1, n}(t)=P\left(T_{1, n}>t\right)=\sum_{j=0}^{n-1} \frac{(R(t))^{j}}{j !} e^{-R(t)}=\bar{\Gamma}_{n}(R(t)), \quad t \geq 0
$$


where $\bar{\Gamma}_{n}$ is the survival function of the gamma distribution with scale parameter 1 and shape parameter $n$; see, for example, Gupta and Kirmani (1988) or Kochar (1996b). The corresponding density function $f_{1, n}$ is given by

$$
f_{1, n}(t)=\gamma_{n}(R(t)) r(t), \quad t \geq 0,
$$

where $\gamma_{n}$ is the density function associated with $\bar{\Gamma}_{n}$. The corresponding hazard rate function $r_{F_{1, \mathrm{n}}}$ is given by

$$
r_{F_{1, n}}(t)=r_{\Gamma_{n}}(R(t)) r(t), \quad t \geq 0
$$

where $r_{\Gamma_{n}}$ is the hazard rate function associated with $\bar{\Gamma}_{n}$. Similarly,

$$
r_{F_{2, \mathrm{n}}}(t)=r_{\Gamma_{\mathrm{n}}}(S(t)) s(t), \quad t \geq 0
$$

If $F \leq_{\mathrm{hr}} G$ then

$$
r_{F_{1, n}}(t)=r_{\Gamma_{n}}(R(t)) r(t) \geq r_{\Gamma_{n}}(S(t)) s(t)=r_{F_{2, n}}(t), \quad t \geq 0
$$

where the inequality follows from $r(t) \geq s(t), R(t) \geq S(t)$, and the fact that the hazard rate function of the gamma $(n)$ distribution is increasing.

The necessity part follows from the fact that $F$ is the distribution function of $T_{1,1}$ and $G$ is the distribution function of $T_{2,1}$.

The next result (Theorem 3.5) gives conditions under which the epoch times of the first process are smaller than the epoch times of the other process in the multivariate likelihood ratio order. This order is defined as follows (see, for example, Section 4.E in [19]). Let $\boldsymbol{X}$ and $\boldsymbol{Y}$ be two $n$-dimensional random vectors with density functions $f_{\boldsymbol{X}}$ and $f_{\boldsymbol{Y}}$, respectively. If

$$
\begin{aligned}
f_{\boldsymbol{X}}\left(x_{1} \wedge y_{1}, x_{2} \wedge y_{2}, \ldots, x_{n} \wedge y_{n}\right) f_{\mathbf{Y}}\left(x_{1} \vee y_{1}, x_{2} \vee y_{2}, \ldots, x_{n} \vee y_{n}\right) \\
\quad \geq f_{\boldsymbol{X}}\left(x_{1}, x_{2}, \ldots, x_{n}\right) f_{\mathbf{Y}}\left(y_{1}, y_{2}, \ldots, y_{n}\right)
\end{aligned}
$$

for all $\left(x_{1}, x_{2}, \ldots, x_{n}\right)$ and $\left(y_{1}, y_{2}, \ldots, y_{n}\right)$ in $\mathbb{R}^{n}$, then we denote $\boldsymbol{X} \leq_{\mathbf{r}} \boldsymbol{Y}$. If the distribution functions of $\boldsymbol{X}$ and $\boldsymbol{Y}$ are $F_{\boldsymbol{X}}$ and $F_{\boldsymbol{Y}}$, respectively, then this relation will sometimes be denoted below by $F_{X} \leq_{l_{r}} F_{Y}$. In the univariate case the relation $F_{X} \leq_{\text {lr }} F_{Y}$ is equivalent to the requirement that $f_{Y} / f_{X}$ is an increasing function. It is known (see Theorems 4.E.4 in [19]) that $\boldsymbol{X} \leq_{\mathbf{l r}} \boldsymbol{Y} \Longrightarrow \boldsymbol{X} \leq_{\mathbf{h r}} \boldsymbol{Y}$. Thus Theorem 3.5 gives a stronger conclusion than Theorem 3.2, but under the additional assumption (3.4). The following lemma is used in the proof of Theorem 3.5 below. The proof of the lemma is straightforward and is therefore omitted. 
Lemma 3.4. Let $F$ and $G$ be two distribution functions with associated hazard rate functions $r$ and $s$. If $F \leq_{\mathrm{hr}} G$, and if

$$
\frac{s(t)}{r(t)} \text { is increasing in } t \geq 0
$$

then $F \leq_{\mathrm{lr}} G$.

Theorem 3.5. Let $F$ and $G$ be distribution functions associated with two nonhomogeneous Poisson processes as described above. If $F \leq_{\mathrm{hr}} G$, and if the hazard rate functions $r$ and $s$ satisfy (3.4), then

$$
\left(T_{1,1}, T_{1,2}, \ldots, T_{1, n}\right) \leq_{\mathrm{lr}}\left(T_{2,1}, T_{2,2}, \ldots, T_{2, n}\right), \quad n \geq 1
$$

Proof. First note that by Lemma 3.4 we have $F \leq_{\mathrm{lr}} G$.

Now, the stated result is obvious for $n=1$. So let $n \geq 2$. The density function of $\left(T_{1,1}, T_{1,2}, \ldots, T_{1, n}\right)$ is

$$
h_{1, n}\left(x_{1}, x_{2}, \ldots, x_{n}\right)=r\left(x_{1}\right) r\left(x_{2}\right) \cdots r\left(x_{n-1}\right) f\left(x_{n}\right) \text { for } x_{1} \leq x_{2} \leq \cdots \leq x_{n} .
$$

Similarly, the density function of $\left(T_{2,1}, T_{2,2}, \ldots, T_{2, n}\right)$ is

$$
h_{2, n}\left(x_{1}, x_{2}, \ldots, x_{n}\right)=s\left(x_{1}\right) s\left(x_{2}\right) \cdots s\left(x_{n-1}\right) g\left(x_{n}\right) \text { for } x_{1} \leq x_{2} \leq \cdots \leq x_{n} .
$$

Consider now $\left(x_{1}, x_{2}, \ldots, x_{n}\right)$ and $\left(y_{1}, y_{2}, \ldots, y_{n}\right)$ such that $x_{1} \leq x_{2} \leq \cdots \leq x_{n}$ and $y_{1} \leq y_{2} \leq \cdots \leq y_{n}$. We want to prove that

$$
\begin{aligned}
r\left(x_{1} \wedge y_{1}\right) r\left(x_{2} \wedge y_{2}\right) & \cdots r\left(x_{n-1} \wedge y_{n-1}\right) f\left(x_{n} \wedge y_{n}\right) \\
& \times s\left(x_{1} \vee y_{1}\right) s\left(x_{2} \vee y_{2}\right) \cdots s\left(x_{n-1} \vee y_{n-1}\right) g\left(x_{n} \vee y_{n}\right) \\
& \geq r\left(x_{1}\right) r\left(x_{2}\right) \cdots r\left(x_{n-1}\right) f\left(x_{n}\right) s\left(y_{1}\right) s\left(y_{2}\right) \cdots s\left(y_{n-1}\right) g\left(y_{n}\right)
\end{aligned}
$$

Let $E=\left\{i \leq n-1: x_{i} \geq y_{i}\right\}$. Then $(3.5)$ reduces to

$$
\left(\prod_{i \in E} r\left(y_{i}\right) s\left(x_{i}\right)\right) f\left(x_{n} \wedge y_{n}\right) g\left(x_{n} \vee y_{n}\right) \geq\left(\prod_{i \in E} r\left(x_{i}\right) s\left(y_{i}\right)\right) f\left(x_{n}\right) g\left(y_{n}\right),
$$

and this follows from (3.4) and $F \leq_{\mathrm{lr}} G$.

Since the order $\leq_{\mathrm{lr}}$ is closed under marginalization (see Theorem 4.E.3(b) in [19]) we get, as a corollary, that if $F \leq_{\mathrm{hr}} G$ and if (3.4) holds then $T_{1, n} \leq_{\mathrm{lr}} T_{2, n}$ for all $n \geq 1$. The following theorem is a variation of this corollary. When one compares the following theorem to the above stated corollary, it should be noticed that (3.4) implies (3.6); see a discussion following the proof of Theorem 3.6. It should also be noticed that $F \leq_{\mathrm{lr}} G$ implies $F \leq_{\mathrm{hr}} G$. 
Theorem 3.6. Let $F$ and $G$ be distribution functions associated with two nonhomogeneous Poisson processes as described above. If $F \leq_{\mathrm{lr}} G$, and if the cumulative hazard functions $R$ and $S$ satisfy

$$
\frac{S(t)}{R(t)} \quad \text { is increasing in } t \geq 0
$$

then

$$
T_{1, n} \leq_{\mathrm{Ir}} T_{2, n}, \quad n \geq 1
$$

Conversely, if (3.7) holds then $F \leq_{\mathrm{Ir}} G$ and (3.6) holds.

Proof. By (2.3) the density function of $T_{1, n}$ is given by

$$
f_{1, n}(t)=f(t) \frac{(R(t))^{n-1}}{(n-1) !}, \quad t \geq 0, \quad n \geq 1
$$

and the density function of $T_{2, n}$ is given by

$$
f_{2, n}(t)=g(t) \frac{(S(t))^{n-1}}{(n-1) !}, \quad t \geq 0, \quad n \geq 1 .
$$

Thus,

$$
\frac{f_{2, n}(t)}{f_{1, n}(t)}=\frac{g(t)}{f(t)}\left(\frac{S(t)}{R(t)}\right)^{n-1}
$$

Now, if $F \leq_{1 \mathrm{r}} G$ and (3.6) holds then $f_{2, n} / f_{1, n}$ is increasing and we get (3.7).

Conversely, suppose that (3.7) holds. Applying (3.7) with $n=1$ we obtain $F \leq_{\mathrm{lr}} G$. In order to obtain (3.6), denote $H \equiv \frac{S}{R}, h \equiv \frac{g}{f}$, and $h_{n} \equiv \frac{g_{n}}{f_{n}}$. First suppose that the $h_{n}$ 's are differentiable. Then, when $n \geq 2$ we have

$$
\overline{h_{n}^{\prime}}(t)=H^{n-2}(t)\left[h^{\prime}(t) H(t)+(n-1) h(t) H^{\prime}(t)\right], \quad t \geq 0 .
$$

If $H$ is not increasing then $H^{\prime}\left(t_{0}\right)<0$ for some $t_{0}$. Therefore, for large enough $n$ we have that $h_{n}^{\prime}\left(t_{0}\right)<0$, and this contradicts (3.7). If the $h_{n}$ 's are not differentiable, then the above argument can be easily modified to obtain the same result.

Sengupta and Deshpande (1994) and Rowell and Siegrist (1998) have shown that (3.4) $\Longrightarrow$ (3.6) (in fact, they treated (3.4) and (3.6) as notions of relative aging of two life distributions). Thus the assumptions in Theorem 3.6 are weaker than the assumptions in Theorem 3.5. It is of interest to note that (3.4) does not imply that $F \leq_{\mathrm{Ir}} G$. In fact, (3.4) does not even 
imply that $F \leq_{\mathrm{hr}} G$. In order to see this, let $r$ be a decreasing hazard rate function such that $r(0+)>1$, for example, $r(t)=t^{-1}$; and let $s(t) \equiv 1$ (that is, the hazard rate function of a standard exponential random variable). Then (3.4) holds, but $r(t)$ is not larger than or equal to $s(t)$ for all $t>0$.

It is also of interest to note that $F \leq_{\text {lr }} G$ does not imply (3.6). In order to see it, let $F$ be the uniform distribution on $[0,1]$, and let $G$ be the gamma(2) distribution. Then

$$
\frac{g(t)}{f(t)}= \begin{cases}t e^{t}, & 0 \leq t \leq 1 \\ \infty, & t>1\end{cases}
$$

and this is increasing in $t$; that is, $F \leq_{\mathrm{Ir}} G$. However, the corresponding $\frac{S(t)}{R(t)}$ is positive when $0 \leq t \leq 1$, and is 0 when $t>1$. Therefore (3.6) does not hold.

The next result gives conditions under which the epoch times of the first process are smaller than the epoch times of the other process in the multivariate mean residual life order. Shaked and Shanthikumar (1991) have given a few equivalent definitions of this order. Let $\boldsymbol{X}=\left(X_{1}, X_{2}, \ldots, X_{n}\right)$ be a nonnegative random vector with a finite mean vector. Consider a possible realization ('history') of $\boldsymbol{X}$ at time $t \geq 0$, that is, an event of the form

$$
h_{t}=\left\{X_{I}=t_{I}, X_{\bar{I}}>t e\right\}, \quad 0 e \leq t_{I} \leq t e, I \subseteq\{1,2, \ldots, n\}
$$

here $\boldsymbol{X}_{I}$ and $\boldsymbol{t}_{I}$ are vectors of dimension $|I|, \boldsymbol{X}_{\bar{I}}$ is a vector of dimension $n-|I|(\bar{I}$ denotes the complement of $I$ in $\{1,2, \ldots, n\})$, and $\boldsymbol{e}$ is a vector of 1 's of a proper dimension. Given a history $h_{t}$ as in (3.8), let $i \in \bar{I}$ (that is, $i$ corresponds to an item that is still alive at time $t$ ). Then the multivariate mean residual life, associated with $i$, at time $t$, is defined as follows:

$$
l_{i \mid I}\left(t \mid \boldsymbol{t}_{I}\right)=E\left[X_{i}-t \mid \boldsymbol{X}_{I}=\boldsymbol{t}_{I}, \boldsymbol{X}_{\bar{I}}>t e\right],
$$

where, of course, $i \in \bar{I}, 0 \boldsymbol{e} \leq \boldsymbol{t}_{I} \leq t e$, and $I \subseteq\{1,2, \ldots, n\}$.

Let $\boldsymbol{X}$ and $\boldsymbol{Y}$ be two $n$-dimensional nonnegative random vectors with associated multivariate mean residual life functions $l_{. \mid}(\cdot \mid \cdot)$ and $m_{. \mid}(\cdot \mid \cdot)$, respectively. For the purpose of this paper we will use the definition of the multivariate mean residual life order as given in (4.F.3) of [19]. There is a mistake there. The equation there should be

$$
l_{i \mid I \cup J}\left(u \mid s_{I}, s_{J}\right) \leq m_{i \mid I}\left(u \mid t_{I}\right) \quad \text { whenever } J \cap I=\emptyset, \mathbf{0} \leq \boldsymbol{s}_{I} \leq \boldsymbol{t}_{I} \leq u e, \text { and } \mathbf{0} \leq \boldsymbol{s}_{J} \leq u e,
$$

where $i \in \overline{I \cup J}$, and $\left(\boldsymbol{s}_{I}, \boldsymbol{s}_{J}\right)$ and $\boldsymbol{t}_{I}$ are possible realizations of the underlying random vectors. When the multivariate mean residual life functions of $\boldsymbol{X}$ and $\boldsymbol{Y}$ satisfy (3.9) we 
will denote this by $\boldsymbol{X} \leq_{\mathrm{mrl}} \boldsymbol{Y}$. If the distribution functions of $\boldsymbol{X}$ and $\boldsymbol{Y}$ are $F_{\boldsymbol{X}}$ and $F_{\boldsymbol{Y}}$, respectively, then this relation will sometimes be denoted below by $F_{\boldsymbol{X}} \leq_{\mathrm{mrl}} F_{\boldsymbol{Y}}$. In the univariate case we have that $X \leq_{\mathrm{mrl}} Y$ if, and only if, $l(u) \leq m(u)$ for all $u \geq 0$, where $l(u)=E[X-u \mid X>u]$ and $m(u)=E[Y-u \mid Y>u]$.

Recall that two univariate random variables $X$ and $Y$, with distribution functions $F$ and $G$, respectively, are said to be ordered in the dispersive order (denoted by $X \leq_{\text {disp }} Y$ or $F \leq_{\text {disp }} G$ ) if $F^{-1}(\beta)-F^{-1}(\alpha) \leq G^{-1}(\beta)-G^{-1}(\alpha)$ whenever $0 \leq \alpha \leq \beta \leq 1$ (see Section 2.B in [19]). See also Proposition 4.3 below for a simple condition which implies $F \leq_{\text {disp }} G$

Theorem 3.7. Let $F$ and $G$ be distribution functions associated with two nonhomogeneous Poisson processes as described above. If $F \leq_{\mathrm{mrl}} G$, and if $F \leq_{\mathrm{disp}} G$, then

$$
\left(T_{1,1}, T_{1,2}, \ldots, T_{1, n}\right) \leq_{\mathrm{mrl}}\left(T_{2,1}, T_{2,2}, \ldots, T_{2, n}\right), \quad n \geq 1
$$

Proof. Let $l_{. \mid}(\cdot \mid \cdot)$ be the multivariate mean residual life functions associated with $\left(T_{1,1}, T_{1,2}\right.$,


$\left(T_{2,1}, T_{2,2}, \ldots, T_{2, n}\right)$. Also, let $l$ be the univariate mean residual life function associated with $F$ and let $m$ be the univariate mean residual life function associated with $G$. Finally, denote the inter-epoch intervals associated with the two processes by $X_{i, j} \equiv T_{i, j}-T_{i, j-1}, i=1,2$, $j \geq 1$. From Proposition 3.10 of Shaked and Szekli (1995) it follows that $X_{1, j} \leq_{\mathbf{s t}} X_{2, j}$ for all $j \geq 1$ (see also Theorem 4.1 below) and therefore

$$
E\left[X_{1, j}\right] \leq E\left[X_{2, j}\right], \quad j \geq 1
$$

Fix an $n \geq 1$. First let us obtain an explicit expression for the right hand side of (3.9). Since $T_{2,1} \leq T_{2,2} \leq \cdots \leq T_{2, n}$ a.s., it follows that $\boldsymbol{t}_{I}$ in (3.9) can be a realization of observations up to time $u$ only if $I$ is of the form $I=\{1,2, \ldots, m\}$ for some $m$. In such a case we have

$$
m_{i \mid I}\left(u \mid \boldsymbol{t}_{I}\right)=\left\{\begin{array}{ll}
m(u), & \text { if } i=m+1 ; \\
m(u)+\sum_{j=m+1}^{i-1} E\left[X_{2, j}\right], & \text { if } i>m+1 ;
\end{array} \quad \text { where } I=\{1,2, \ldots, m\} .\right.
$$

Next, let us obtain an explicit expression for the left hand side of (3.9). Since $T_{1,1} \leq T_{1,2} \leq$ $\cdots \leq T_{1, n}$ a.s., we see that when $I=\{1,2, \ldots, m\}$ then $\left(s_{I}, s_{J}\right)$ in (3.9) can be a realization of observations up to time $u$ only if $J$ is of the form $J=\{m+1, m+2, \ldots, k\}$ for some 
$k \geq m$. In such a case we have

$$
l_{i \mid I \cup J}\left(u \mid s_{I}, s_{J}\right)= \begin{cases}l(u), & \text { if } i=k+1 \\ l(u)+\sum_{j=k+1}^{i-1} E\left[X_{1, j}\right], & \text { if } i>k+1\end{cases}
$$

where $I=\{1,2, \ldots, m\}$ and $J=\{m+1, m+2, \ldots, k\}$.

Since $i$ in (3.9) must satisfy $i \in \overline{I U J}$ (that is, $i>k$ ), we see that if $k>m$ then using (3.10) and $l(u) \leq m(u)$ we get

$$
\begin{aligned}
& l_{i \mid I \cup J}\left(u \mid s_{I}, s_{J}\right)=l(u) \leq m(u)+\sum_{j=m+1}^{i-1} E\left[X_{2, j}\right]=m_{i \mid I}\left(u \mid \boldsymbol{t}_{I}\right) \quad \text { if } i=k+1 ; \\
& l_{i \mid I \cup J}\left(u \mid \boldsymbol{s}_{I}, \boldsymbol{s}_{J}\right)=l(u)+\sum_{j=k+1}^{i-1} E\left[X_{1, j}\right] \leq m(u)+\sum_{j=m+1}^{i-1} E\left[X_{2, j}\right]=m_{i \mid I}\left(u \mid \boldsymbol{t}_{I}\right) \quad \text { if } i>k+1
\end{aligned}
$$

so (3.9) holds. If $k=m$ (that is, $J=\emptyset$ ) then

$$
\begin{aligned}
& l_{i \mid I \cup J}\left(u \mid s_{I}, s_{J}\right)=l(u) \leq m(u)=m_{i \mid I}\left(u \mid \boldsymbol{t}_{I}\right) \quad \text { if } i=k+1 ; \\
& l_{i \mid I \cup J}\left(u \mid \boldsymbol{s}_{I}, \boldsymbol{s}_{J}\right)=l(u)+\sum_{j=k+1}^{i-1} E\left[X_{1, j}\right] \leq m(u)+\sum_{j=k+1}^{i-1} E\left[X_{2, j}\right]=m_{i \mid I}\left(u \mid \boldsymbol{t}_{I}\right) \quad \text { if } i>k+1 ;
\end{aligned}
$$

so (3.9) holds in this case too.

Before we close this subsection it is worthwhile to mention that if $F$ and $G$ are distribution functions associated with two nonhomogeneous Poisson processes as described above, then $F \leq_{\mathrm{c}}\left[\leq_{*}, \leq_{\mathrm{su}}\right] G$ if, and only if, $T_{1, n} \leq_{\mathrm{c}}\left[\leq_{*}, \leq_{\mathrm{su}}\right] T_{2, n}$, where $\leq_{\mathrm{c}}, \leq_{*}$ and $\leq_{\mathrm{su}}$ are the transform orders described in Section 3.C of [19]; this is Theorem 2 of Gupta and Kirmani (1988). Using the idea of their proof we also obtain the following result.

Theorem 3.8. Let $F$ and $G$ be distribution functions associated with two nonhomogeneous Poisson processes as described above. Then $F \leq_{\operatorname{disp}} G$ if, and only if, $T_{1, n} \leq_{\operatorname{disp}} T_{2, n}$ for all $n \geq 1$.

Proof. Fix an $n \geq 1$, and denote by $F_{1, n}$ and $F_{2, n}$ the distribution functions of $T_{1, n}$ and $T_{2, n}$, respectively. Recall from (3.3) that

$$
F_{1, n}(t)=\psi_{n}(F(t)) \quad \text { and } \quad F_{2, n}(t)=\psi_{n}(G(t)),
$$

where $\psi(u) \equiv \Gamma_{n}(-\log (1-u)), u \in[0,1]$. Therefore,

$$
F_{2, n}^{-1}\left(F_{1, n}(t)\right)-t=\left(\psi_{n}(G)\right)^{-1}\left(\psi_{n}(F(t))\right)-t=G^{-1}(F(t))-t, \quad t \geq 0 .
$$

Thus, from (2.B.6) in [19] it is seen that $F \leq_{\text {disp }} G$ if, and only if, $T_{1, n} \leq_{\text {disp }} T_{2, n}$. 


\subsection{Nonhomogeneous birth processes}

Consider two nonhomogeneous birth processes. Let the first one be associated with a sequence $\left\{Y_{1, n}, n=1,2, \ldots\right\}$ of independent absolutely continuous nonnegative random variables with density, distribution, and hazard rate functions $k_{1, n}, K_{1, n}$, and $r_{1, n}$, respectively. Let the second one be associated with a sequence $\left\{Y_{2, n}, n=1,2, \ldots\right\}$ of independent absolutely continuous nonnegative random variables with density, distribution, and hazard rate functions $k_{2, n}, K_{2, n}$, and $r_{2, n}$, respectively. For $i=1,2$, define the epoch times of the two processes by

$$
\begin{aligned}
& T_{i, 1}={ }_{\mathrm{st}} Y_{i, 1}, \\
& T_{i, n}={ }_{\text {st }}\left[Y_{i, n} \mid Y_{i, n}>T_{i, n-1}\right], \quad n \geq 2,
\end{aligned}
$$

as in (2.5) and (2.6). In this subsection we derive some extensions and analogs of the results in the previous subsection.

The first result gives conditions under which the epoch times of the two nonhomogeneous birth processes are ordered according to the usual stochastic order. This result may be compared with Theorem 3.1. The following result can be proven using some general ideas from Shaked and Szekli (1995), but we give here a simpler direct proof of it.

Theorem 3.9. Let $T_{i, n}$ be defined as in (3.11) and (3.12). If $Y_{1,1} \leq_{\mathrm{st}} Y_{2,1}$ and if $Y_{1, j} \leq_{\mathrm{hr}} Y_{2, j}$ for $j \geq 2$ then

$$
\left(T_{1,1}, T_{1,2}, \ldots, T_{1, n}\right) \leq_{\mathrm{st}}\left(T_{2,1}, T_{2,2}, \ldots, T_{2, n}\right), \quad n \geq 1
$$

Proof. We will apply Theorem 4.B.4 in [19]. Note that for $j \geq 2$ we have

$$
\left[T_{1, j} \mid T_{1,1}=t_{1}, T_{1,2}=t_{2}, \ldots, T_{1, j-1}=t_{j-1}\right]=_{\mathbf{s t}}\left[Y_{1, j} \mid Y_{1, j}>t_{j-1}\right]
$$

and this is stochastically increasing in $t_{j-1}$ (see Theorem 1.A.11 in [19]). Therefore $\left(T_{1,1}, T_{1,2}\right.$, $\ldots, T_{1, n}$ ) is CIS (conditionally increasing in sequence, see [19, page 117]). Next note that

$$
\begin{aligned}
& {\left[T_{1, j} \mid T_{1,1}=t_{1}, T_{1,2}=\right.}\left.t_{2}, \ldots, T_{1, j-1}=t_{j-1}\right]==_{\text {st }}\left[Y_{1, j} \mid Y_{1, j}>t_{j-1}\right] \\
& \leq_{\text {st }}\left[Y_{2, j} \mid Y_{2, j}>t_{j-1}\right]==_{\text {st }}\left[T_{2, j} \mid T_{2,1}=t_{1}, T_{2,2}=t_{2}, \ldots, T_{2, j-1}=t_{j-1}\right]
\end{aligned}
$$

where the inequality, which is equivalent to

$$
\frac{\bar{K}_{1, j}(u)}{\bar{K}_{1, j}\left(t_{j-1}\right)} \leq \frac{\bar{K}_{2, j}(u)}{\bar{K}_{2, j}\left(t_{j-1}\right)}, \quad u \geq t_{j-1},
$$

follows from $Y_{1, j} \leq_{\mathrm{hr}} Y_{2, j}$. Thus (3.13) follows from Theorem 4.B.4 in [19]. 
The next result is an extension of Theorem 3.2 to nonhomogeneous birth processes.

Theorem 3.10. Let $T_{i, n}$ be defined as in (3.11) and (3.12). If $Y_{1, j} \leq_{\mathrm{hr}} Y_{2, j}$ for $j \geq 1$ then $\left(T_{1,1}, T_{1,2}, \ldots, T_{1, n}\right) \leq_{\mathrm{hr}}\left(T_{2,1}, T_{2,2}, \ldots, T_{2, n}\right)$ for all $n \geq 1$.

Proof. The proof is similar to the proof of Theorem 3.2. Fix an $n \geq 1$. Let $\eta \cdot . \cdot(\cdot \mid \cdot)$ be the multivariate conditional hazard rate functions associated with $\left(T_{1,1}, T_{1,2}, \ldots, T_{1, n}\right)$ and let $\lambda_{\text {.|. }}(\cdot \mid \cdot)$ be the multivariate conditional hazard rate functions associated with $\left(T_{2,1}, T_{2,2}, \ldots, T_{2, n}\right)$.

In order to obtain an explicit expression for the right hand side of (3.2), we first notice, as in the proof of Theorem 3.2 , that $I$ there must be of the form $I=\{1,2, \ldots, m\}$ for some $m$. Then we have

$$
\lambda_{i \mid I}\left(u \mid \boldsymbol{t}_{I}\right)=\left\{\begin{array}{ll}
r_{2, m+1}(u), & \text { if } i=m+1 ; \\
0, & \text { if } i>m+1
\end{array} \quad \text { where } I=\{1,2, \ldots, m\}\right.
$$

Similarly, in the left hand side of $(3.2)$ we must have $I=\{1,2, \ldots, m\}$ and $J=\{m+1, m+$ $2, \ldots, k\}$ for some $k \geq m$. Then we have

$$
\begin{aligned}
\eta_{i \mid I \cup J}\left(u \mid s_{I}, s_{J}\right)= \begin{cases}r_{1, k+1}(u), & \text { if } i=k+1 \\
0, & \text { if } i>k+1\end{cases} \\
\text { where } I=\{1,2, \ldots, m\} \text { and } J=\{m+1, m+2, \ldots, k\} .
\end{aligned}
$$

The rest of the proof follows the lines of the proof of Theorem 3.2.

The next result is an extension of Theorem 3.5 to nonhomogeneous birth processes. At a first glance condition (3.14) in the following theorem looks restrictive, however in many applications (see Section 5 below) the hazard rate functions $r_{1,1}, r_{1,2}, \ldots$ are proportional, and the hazard rate functions $r_{2,1}, r_{2,2}, \ldots$ are also proportional, and then $(3.14)$ can often be verified.

Theorem 3.11. Let $T_{i, n}$ be defined as in (3.11) and (3.12). If $Y_{1, j} \leq_{\mathrm{hr}} Y_{2, j}$, and if $r_{2, j} / r_{1, j}$ is increasing, and if

$$
r_{2, j+1}(t)-r_{2, j}(t) \geq r_{1, j+1}(t)-r_{1, j}(t), \quad t \geq 0
$$

for $j \geq 1$, then $\left(T_{1,1}, T_{1,2}, \ldots, T_{1, n}\right) \leq_{\operatorname{lr}}\left(T_{2,1}, T_{2,2}, \ldots, T_{2, n}\right)$ for all $n \geq 1$. 
Proof. First note that by Lemma 3.4 we have $Y_{1, j} \leq_{\mathrm{Ir}} Y_{2, j}, j \geq 1$.

Now, the stated result is obvious for $n=1$. So let $n \geq 2$. The density function $h_{1, n}$ of $\left(T_{1,1}, T_{1,2}, \ldots, T_{1, n}\right)$ is given by

$$
h_{1, n}\left(x_{1}, x_{2}, \ldots, x_{n}\right)=\prod_{i=1}^{n-1} \frac{k_{1, i}\left(x_{i}\right)}{\bar{K}_{1, i+1}\left(x_{i}\right)} k_{1, n}\left(x_{n}\right) \quad \text { for } x_{1} \leq x_{2} \leq \cdots \leq x_{n} .
$$

Similarly, the density function $h_{2, n}$ of $\left(T_{2,1}, T_{2,2}, \ldots, T_{2, n}\right)$ is given by

$$
h_{2, n}\left(x_{1}, x_{2}, \ldots, x_{n}\right)=\prod_{i=1}^{n-1} \frac{k_{2, i}\left(x_{i}\right)}{\bar{K}_{2, i+1}\left(x_{i}\right)} k_{2, n}\left(x_{n}\right) \text { for } x_{1} \leq x_{2} \leq \cdots \leq x_{n} .
$$

Note that condition (3.14) can be written as

$$
\frac{\bar{K}_{2, j}(t) \bar{K}_{1, j+1}(t)}{\bar{K}_{2, j+1}(t) \bar{K}_{1, j}(t)} \text { is increasing in } t \geq 0 .
$$

Consider now $\left(x_{1}, x_{2}, \ldots, x_{n}\right)$ and $\left(y_{1}, y_{2}, \ldots, y_{n}\right)$ such that $x_{1} \leq x_{2} \leq \cdots \leq x_{n}$ and $y_{1} \leq y_{2} \leq \cdots \leq y_{n}$. We want to prove that

$$
\begin{aligned}
\prod_{i=1}^{n-1} \frac{k_{1, i}\left(x_{i} \wedge y_{i}\right)}{\bar{K}_{1, i+1}\left(x_{i} \wedge y_{i}\right)} k_{1, n}\left(x_{n} \wedge y_{n}\right) \prod_{i=1}^{n-1} \frac{k_{2, i}\left(x_{i} \vee y_{i}\right)}{\bar{K}_{2, i+1}\left(x_{i} \vee y_{i}\right)} k_{2, n}\left(x_{n} \vee y_{n}\right) \\
\geq \prod_{i=1}^{n-1} \frac{k_{1, i}\left(x_{i}\right)}{\bar{K}_{1, i+1}\left(x_{i}\right)} k_{1, n}\left(x_{n}\right) \prod_{i=1}^{n-1} \frac{k_{2, i}\left(y_{i}\right)}{\bar{K}_{2, i+1}\left(y_{i}\right)} k_{2, n}\left(y_{n}\right)
\end{aligned}
$$

Let $E=\left\{i \leq n-1: x_{i} \geq y_{i}\right\}$. Then (3.16) reduces to

$$
\begin{aligned}
\left(\prod_{i \in E} r_{1, i}\left(y_{i}\right) \frac{\bar{K}_{1, i}\left(y_{i}\right)}{\bar{K}_{1, i+1}\left(y_{i}\right)} r_{2, i}\left(x_{i}\right) \frac{\bar{K}_{2, i}\left(x_{i}\right)}{\bar{K}_{2, i+1}\left(x_{i}\right)}\right) k_{1, n}\left(x_{n} \wedge y_{n}\right) k_{2, n}\left(x_{n} \vee y_{n}\right) \\
\geq\left(\prod_{i \in E} r_{1, i}\left(x_{i}\right) \frac{\bar{K}_{1, i}\left(x_{i}\right)}{\bar{K}_{1, i+1}\left(x_{i}\right)} r_{2, i}\left(y_{i}\right) \frac{\bar{K}_{2, i}\left(y_{i}\right)}{\bar{K}_{2, i+1}\left(y_{i}\right)}\right) k_{1, n}\left(x_{n}\right) k_{2, n}\left(y_{n}\right),
\end{aligned}
$$

and this follows from the monotonicity of $r_{2, j} / r_{1, j}$, from (3.15), and from $Y_{1, j} \leq_{\mathrm{lr}} Y_{2, j}$.

\section{Stochastic Comparisons of Inter-Epoch Intervals}

\subsection{Nonhomogeneous Poisson processes}

As in Subsection 3.1, consider two nonhomogeneous Poisson processes with intensity functions $r$ and $s$, respectively, and with associated density functions (see (2.2)) $f$ and $g$, respectively, and associated distribution functions $F$ and $G$, respectively, and associated cumulative 
hazard functions $R$ and $S$, respectively. Let $T_{1, j}$ and $T_{2, j}$ be as defined in Subsection 3.1. Let the inter-epoch intervals of the first nonhomogeneous Poisson process be denoted by $X_{1, i}=T_{1, i}-T_{1, i-1}, i=1,2, \ldots$, with $T_{1,0} \equiv 0$, and similarly, let the inter-epoch intervals of the other nonhomogeneous Poisson process be denoted by $X_{2, i}=T_{2, i}-T_{2, i-1}, i=1,2, \ldots$, with $T_{2,0} \equiv 0$.

In this subsection we derive some results which stochastically compare vectors of $X_{1, j}$ 's with vectors of $X_{2, j}$ 's.

The first result gives conditions under which the inter-epoch intervals of the first process are smaller than the inter-epoch intervals of the other process in the usual stochastic order sense. It is essentially a restatement of Proposition 3.10 of Shaked and Szekli (1995).

Theorem 4.1. Let $F$ and $G$ be distribution functions associated with two nonhomogeneous Poisson processes as described above. If $F \leq_{\operatorname{disp}} G$ then

$$
\left(X_{1,1}, X_{1,2}, \ldots, X_{1, n}\right) \leq_{\text {st }}\left(X_{2,1}, X_{2,2}, \ldots, X_{2, n}\right), \quad n \geq 1
$$

Roughly speaking, inequality (4.1) for $n=\infty$ is denoted in Shaked and Szekli (1995) as $N_{1} \geq_{\text {st- } \infty} N_{2}$, where $N_{1}$ and $N_{2}$ are the underlying nonhomogeneous Poisson processes.

A similar result which is worth mentioning is the following; it follows from Theorem 2.7 of Shaked and Szekli (1995).

Theorem 4.2. Let $r$ and $s$ be intensity functions associated with two nonhomogeneous Poisson processes as described above. If

$$
r(u) \geq s(u+x), \quad u \geq 0, x \geq 0
$$

then

$$
\left(X_{1,1}, X_{1,2}, \ldots, X_{1, n}\right) \leq_{\text {st }}\left(X_{2,1}, X_{2,2}, \ldots, X_{2, n}\right), \quad n \geq 1
$$

Note that (4.2) holds if $F \leq_{\mathrm{hr}} G$ and if $r$ or $s$ is decreasing (that is, $F$ or $G$ is DFR). Thus, Theorem 4.2 is a stronger result than Theorem 8 of Gupta and Kirmani (1988) or Theorem 4.4 of Kochar (1996a). In fact we have the following relationship among the conditions of Theorems 4.1 and 4.2 .

Proposition 4.3. Let $F$ and $G$ be two 'distribution functions with respective hazard rate functions $r$ and s. If (4.2) holds then $F \leq_{\operatorname{disp}} G$. 
Proof. Condition (4.2) implies that $r(u) \geq s(u)$, that is, $F \leq_{\mathrm{hr}} G$. This, in turn, implies $F \leq_{\text {st }} G$, and therefore $F^{-1}(\alpha) \leq G^{-1}(\alpha)$ for all $\alpha \in(0,1)$.

Now, (4.2) therefore gives $r\left(F^{-1}(\alpha)\right) \geq s\left(G^{-1}(\alpha)\right)$ for all $\alpha \in(0,1)$, which is equivalent to $F \leq_{\text {disp }} G$ by $(2 . B .8)$ in [19].

From Proposition 4.3 it is seen that Theorem 4.2 follows from Theorem 4.1. Proposition 4.3 also strengthens a result of Bartoszewicz (1985) and of Bagai and Kochar (1986) which is stated as Theorem 2.B.13(a) in [19]. This is so because if $F \leq_{\mathrm{hr}} G$ and if $r$ or $s$ is decreasing then (4.2) holds.

Condition (4.2) defines what can be called a 'shifted hazard rate order' in the spirit of Shanthikumar and Yao (1986) who defined a 'shifted likelihood ratio order.' However, it should be noticed that whereas (4.2) is the same as $X \leq_{\mathrm{hr}}[Y-x \mid Y>x]$ for all $x \geq 0$, where $X$ and $Y$ have the hazard rates functions $r$ and $s$, respectively, the condition of Shanthikumar and Yao is the same as $[X-x \mid X>x] \leq_{\operatorname{lr}} Y$ for all $x \geq 0$.

The next result gives conditions under which each inter-epoch of the first process is smaller than the corresponding inter-epoch of the other process in the hazard rate order.

Theorem 4.4. Let $F$ and $G$ be distribution functions associated with two nonhomogeneous Poisson processes as described above, with corresponding hazard rate functions $r$ and $s$, and with corresponding cumulative hazard functions $R$ and $S$, respectively. If $F \leq_{\mathrm{hr}} G$, and if $\bar{F}$ and $\bar{G}$ are logconvex (that is, DFR), and if (3.4) holds, then $X_{1, n} \leq_{\mathrm{hr}} X_{2, n}$ for each $n \geq 1$.

Proof. For the purpose of this proof we denote $F$ by $F_{1}, G$ by $F_{2}, r$ by $r_{1}, s$ by $r_{2}, R$ by $R_{1}$, and $S$ by $R_{2}$. Let $\bar{G}_{i, n}$ denote the survival function of $X_{i, n}, i=1,2$. The stated result is obvious for $n=1$, so let us fix an $n \geq 2$. Then, from (2.4) we obtain

$$
\bar{G}_{i, n}^{-}(t)=\int_{0}^{\infty} r_{i}(s) \frac{R_{i}^{n-2}(s)}{(n-2) !} \bar{F}_{i}(s+t) d s, \quad t \geq 0, i \in\{1,2\} .
$$

Condition (3.4) means that

$$
r_{i}(t) \text { is } \mathrm{TP}_{2} \text { (totally positive of order } 2 \text { ) in }(i, t)
$$

Condition (3.4) also implies that $\frac{R_{2}(t)}{R_{1}(t)}$ is increasing in $t \geq 0$; that is, $R_{i}(t)$ is $\operatorname{TP}_{2}$ in $(i, t)$. Since a product of $\mathrm{TP}_{2}$ kernels is $\mathrm{TP}_{2}$ we get that

$$
r_{i}(t) \frac{R_{i}^{n-2}(t)}{(n-2) !} \text { is } \mathrm{TP}_{2} \text { in }(i, t)
$$


The assumption $F_{1} \leq_{\mathrm{hr}} F_{2}$ implies that

$$
\bar{F}_{i}(s+t) \text { is } \mathrm{TP}_{2} \text { in }(i, s) \text { and in }(i, t) .
$$

Finally, the logconvexity of $\bar{F}_{1}$ and of $\bar{F}_{2}$ means that

$$
\bar{F}_{i}(s+t) \text { is } \mathrm{TP}_{2} \text { in }(s, t) \text {. }
$$

Thus, by Theorem 5.1 in page 123 of Karlin (1968), we get that $\bar{G}_{i, n}(t)$ is $\mathrm{TP}_{2}$ in $(i, t)$; that is, $X_{1, n} \leq_{\mathrm{hr}} X_{2, n}$.

The next result gives conditions under which the inter-epoch intervals of the first process are smaller than the inter-epoch intervals of the other process in the multivariate likelihood ratio order.

Theorem 4.5. Let $F$ and $G$ be distribution functions associated with two nonhomogeneous Poisson processes as described above, with corresponding density functions $f$ and $g$, and with corresponding hazard rate functions $r$ and $s$. If $F \leq_{\mathrm{hr}} G$, and if $f$ and $r$ are logconvex or $g$ and $s$ are logconvex, and if (3.4) holds, then

$$
\left(X_{1,1}, X_{1,2}, \ldots, X_{1, n}\right) \leq \operatorname{lr}\left(X_{2,1}, X_{2,2}, \ldots, X_{2, n}\right), \quad n \geq 1
$$

Proof. First note that by Lemma 3.4 we have $F \leq_{\mathrm{lr}} G$.

We will give the proof when $f$ and $r$ are logconvex; the proof when $g$ and $s$ are logconvex is similar. Note that the logconvexity of $f$ and $r$ implies that $f$ and $r$ are positive over $(0, \infty)$. The result is obvious for $n=1$, thus let us fix an $n \geq 2$. The density function $\ell_{1, n}$ of $\left(X_{1,1}, X_{1,2}, \ldots, X_{1, n}\right)$ is given by

$$
\ell_{1, n}\left(x_{1}, \ldots, x_{n}\right)=\prod_{j=1}^{n-1} r\left(x_{1}+\cdots+x_{j}\right) f\left(x_{1}+\cdots+x_{n}\right), \quad x_{k} \geq 0, k=1, \ldots, n .
$$

The density function $\ell_{2, n}$ of $\left(X_{2,1}, X_{2,2}, \ldots, X_{2, n}\right)$ is given by

$$
\ell_{2, n}\left(x_{1}, \ldots, x_{n}\right)=\prod_{j=1}^{n-1} s\left(x_{1}+\cdots+x_{j}\right) g\left(x_{1}+\cdots+x_{n}\right), \quad x_{k} \geq 0, k=1, \ldots, n .
$$

The logconvexity of $f$ implies that

$$
f\left(x_{1} \vee y_{1}+\cdots+x_{n} \vee y_{n}\right) f\left(x_{1} \wedge y_{1}+\cdots+x_{n} \wedge y_{n}\right) \geq f\left(x_{1}+\cdots+x_{n}\right) f\left(y_{1}+\cdots+y_{n}\right)
$$


for all $x_{k} \geq 0$ and $y_{k} \geq 0, k=1, \ldots, n$. Similarly, the logconvexity of $r$ implies, for $j=1, \ldots, n-1$, that

$$
r\left(x_{1} \vee y_{1}+\cdots+x_{j} \vee y_{j}\right) r\left(x_{1} \wedge y_{1}+\cdots+x_{j} \wedge y_{j}\right) \geq r\left(x_{1}+\cdots+x_{j}\right) r\left(y_{1}+\cdots+y_{j}\right)
$$

for all $x_{k} \geq 0$ and $y_{k} \geq 0, k=1, \ldots, j$. Therefore

$$
\begin{aligned}
& \ell_{1, n}\left(x_{1} \wedge\right.\left.y_{1}, \ldots, x_{n} \wedge y_{n}\right) \ell_{2, n}\left(x_{1} \vee y_{1}, \ldots, x_{n} \vee y_{n}\right) \\
&= \prod_{j=1}^{n-1} r\left(x_{1} \wedge y_{1}+\cdots+x_{j} \wedge y_{j}\right) f\left(x_{1} \wedge y_{1}+\cdots+x_{n} \wedge y_{n}\right) \\
& \times \prod_{j=1}^{n-1} s\left(x_{1} \vee y_{1}+\cdots+x_{j} \vee y_{j}\right) g\left(x_{1} \vee y_{1}+\cdots+x_{n} \vee y_{n}\right) \\
& \geq \prod_{j=1}^{n-1} \frac{s\left(x_{1} \vee y_{1}+\cdots+x_{j} \vee y_{j}\right)}{r\left(x_{1} \vee y_{1}+\cdots+x_{j} \vee y_{j}\right)} r\left(x_{1}+\cdots+x_{j}\right) r\left(y_{1}+\cdots+y_{j}\right) \\
& \times \frac{g\left(x_{1} \vee y_{1}+\cdots+x_{n} \vee y_{n}\right)}{f\left(x_{1} \vee y_{1}+\cdots+x_{n} \vee y_{n}\right)} f\left(x_{1}+\cdots+x_{n}\right) f\left(y_{1}+\cdots+y_{n}\right) \\
& \geq \prod_{j=1}^{n-1} \frac{s\left(y_{1}+\cdots+y_{j}\right)}{r\left(y_{1}+\cdots+y_{j}\right)} r\left(x_{1}+\cdots+x_{j}\right) r\left(y_{1}+\cdots+y_{j}\right) \\
& \times \frac{g\left(y_{1}+\cdots+y_{n}\right)}{f\left(y_{1}+\cdots+y_{n}\right)} f\left(x_{1}+\cdots+x_{n}\right) f\left(y_{1}+\cdots+y_{n}\right) \\
&=
\end{aligned}
$$

where the first inequality follows from (4.6) and (4.7), and the second inequality follows from (3.4) and from $F \leq \operatorname{lr} G$ (that is, $g / f$ is increasing). This gives the stated result.

In light of the conditions in Theorem 4.5 the following question is of interest: Let $f$ be a density function of a nonnegative random variable, and let $r$ be the corresponding hazard rate function. Does the logconvexity of $f$ imply the logconvexity of $r$, and vice versa? It turns out that neither is the case. First consider the hazard rate function

$$
r(t)=e^{t}, \quad t \geq 0
$$

Here $\log r$ is linear, so it is logconvex. The corresponding density function is given by

$$
f(t)=e^{1+t-e^{\imath}}, \quad t \geq 0,
$$

and a computation of the second derivative shows that $f$ here is strictly logconcave (see Pellerey, Shaked and Zinn (1999)), and thus it is not logconvex. In order to see that ' $f$ is 
logconvex' does not imply that ' $r$ is logconvex,' consider the hazard rate function

$$
r(t)=\frac{1}{t+3}-\frac{1}{(t+3)^{2}}=\frac{t+2}{(t+3)^{2}}, \quad t \geq 0
$$

This is indeed a hazard rate function since it is nonnegative, and it integrates to $\infty$. A straightforward computation shows that $\left(d^{2} / d t^{2}\right) \log r(t)<0$ for $0<t<2 \sqrt{2}-2$. Therefore $r$ is not logconvex. The corresponding density function is

$$
f(t)=\frac{3(t+2)}{(t+3)^{3}} \exp \left\{\frac{t}{3(t+3)}\right\}, \quad t \geq 0 .
$$

A straightforward computation gives

$$
\frac{d^{2}}{d t^{2}} \log f(t)=\frac{2 t^{3}+10 t^{2}+13 t+1}{(t+3)^{3}(t+2)^{2}}
$$

and this is positive for all $t \geq 0$. Thus $f$ is logconvex.

Since the multivariate likelihood ratio order is closed under marginalization (see Theorem 4.E.3(b) in [19]), we get the following result as a corollary of Theorem 4.5.

Corollary 4.6. Let $F$ and $G$ be distribution functions associated with two nonhomogeneous Poisson processes as described above, with corresponding density functions $f$ and $g$, and with corresponding hazard rate functions $r$ and $s$. If $F \leq_{\mathrm{hr}} G$, and if $f$ and $r$ are logconvex or $g$ and $s$ are logconvex, and if (3.4) holds, then $X_{1, n} \leq_{1 \mathrm{r}} X_{2, n}$ for all $n \geq 1$.

The next result gives different conditions under which each inter-epoch of the first process is smaller than the corresponding inter-epoch of the other process in the likelihood ratio order.

Theorem 4.7. Let $f$ and $g$ be density functions associated with two nonhomogeneous Poisson processes as described above, with corresponding hazard rate functions $r$ and $s$, and with corresponding cumulative hazard functions $R$ and $S$, respectively. If $F \leq_{\mathrm{hr}} G$, and if $f$ and $g$ are logconvex, and if (3.4) holds, then $X_{1, n} \leq_{1 \mathrm{r}} X_{2, n}$ for each $n \geq 1$.

Proof. First note that by Lemma 3.4 we have $F \leq_{\text {Ir }} G$.

For the purpose of this proof we denote $f$ by $f_{1}, g$ by $f_{2}, r$ by $r_{1}, s$ by $r_{2}, R$ by $R_{1}$, and $S$ by $R_{2}$. Let $g_{i, n}$ denote the density function of $X_{i, n}, i=1,2$. The stated result is obvious for $n=1$, so let us fix an $n \geq 2$. From (2.4) we obtain

$$
g_{i, n}(t)=\int_{0}^{\infty} r_{i}(s) \frac{R_{i}^{n-2}(s)}{(n-2) !} f_{i}(s+t) d s, \quad t \geq 0, i=1,2 .
$$


As in the proof of Theorem 4.4, we have that

$$
r_{i}(t) \frac{R_{i}^{n-2}(t)}{(n-2) !} \text { is } \mathrm{TP}_{2} \text { in }(i, t) .
$$

The assumption $F_{1} \leq_{\mathrm{lr}} F_{2}$ implies that

$$
f_{i}(s+t) \text { is } \mathrm{TP}_{2} \text { in }(i, s) \text { and in }(i, t) .
$$

Finally, the logconvexity of $f_{1}$ and of $f_{2}$ means that

$$
f_{i}(s+t) \text { is } \mathrm{TP}_{2} \text { in }(s, t) \text {. }
$$

Thus, by Theorem 5.1 in page 123 of Karlin (1968), we get that, $g_{i, n}(t)$ is $\mathrm{TP}_{2}$ in $(i, t)$; that is, $X_{1, n} \leq_{\mathrm{lr}} X_{2, n}$.

The next result gives conditions under which each inter-epoch of the first process is smaller than the corresponding inter-epoch of the other process in the mean residual life order. Recall that a distribution function $F$ is said to be IMRL (increasing mean residual life) if the mean residual life at time $t$, defined as $\left(\int_{t}^{\infty} \bar{F}(u) d u\right) / \bar{F}(t)$, is increasing in $t \geq 0$ when the ratio is well defined.

Theorem 4.8. Let $F$ and $G$ be distribution functions associated with two nonhomogeneous Poisson processes as described above, with corresponding hazard rate functions $r$ and $s$, and with corresponding cumulative hazard functions $R$ and $S$, respectively. If $F \leq_{\mathrm{mrl}} G$, and if $\bar{F}$ and $\bar{G}$ are IMRL, and if (3.4) holds, then $X_{1, n} \leq_{\mathrm{mrl}} X_{2, n}$ for each $n \geq 1$.

Proof. As in the proof of Theorem 4.4, we denote here $F$ by $F_{1}, G$ by $F_{2}, r$ by $r_{1}, s$ by $r_{2}$, $R$ by $R_{1}$, and $S$ by $R_{2}$. The stated result is obvious for $n=1$, so let us fix an $n \geq 2$. The survival function $\bar{G}_{i, n}$ of $X_{i, n}, i=1,2$, is given in (4.3). From (1.D.3) in [19] it is seen that the stated result is equivalent to

$$
\int_{t}^{\infty} \bar{G}_{i, n}(x) d x \quad \text { is } \mathrm{TP}_{2} \text { in }(i, t)
$$

that is, to

$$
\int_{s=0}^{\infty} r_{i}(s) \frac{R_{i}^{n-2}(s)}{(n-2) !} \int_{u=s+t}^{\infty} \bar{F}_{i}(u) d u d s \quad \text { is } \mathrm{TP}_{2} \text { in }(i, t)
$$

Now, from the proof of Theorem 4.4 we know that (3.4) implies that $r_{i}(s) \frac{R_{i}^{n-2}(s)}{(n-2) !}$ is $\mathrm{TP}_{2}$ in $(i, s)$. The assumption $F_{1} \leq_{\mathrm{mrl}} F_{2}$ means that

$$
\int_{u=s+t}^{\infty} \bar{F}_{i}(u) d u \quad \text { is } \mathrm{TP}_{2} \text { in }(i, s) \text { and in }(i, t)
$$


Finally, the assumption that $F_{i}$ is IMRL means that

$$
\int_{u=s+t}^{\infty} \bar{F}_{i}(u) d u \quad \text { is } \mathrm{TP}_{2} \text { in }(s, t)
$$

Thus (4.8) follows from Theorem 5.1 in page 123 of Karlin (1968).

\subsection{Nonhomogeneous birth processes}

In this subsection, as in Subsection 3.2, we consider two nonhomogeneous birth processes. Let the first one be associated with a sequence $\left\{Y_{1, n}, n=1,2, \ldots\right\}$ of independent absolutely continuous nonnegative random variables, and let the second one be associated with a sequence $\left\{Y_{2, n}, n=1,2, \ldots\right\}$ of independent absolutely continuous nonnegative random variables. The notation of Subsection 3.2 will be used here. In particular, the epoch times $T_{i, n}$ associated with the two processes are defined in (3.11) and (3.12). The associated inter-epoch intervals will be defined as

$$
\begin{aligned}
& X_{i, 1}={ }_{\text {st }} Y_{i, 1}, \\
& X_{i, n}={ }_{\text {st }}\left[Y_{i, n}-T_{i, n-1} \mid Y_{i, n}>T_{i, n-1}\right], \quad n \geq 2,
\end{aligned}
$$

The density function $\ell_{1, n}$ of $\left(X_{1,1}, X_{1,2}, \ldots, X_{1, n}\right)$ is an extension of (4.4) and is given by

$$
\ell_{1, n}\left(x_{1}, \ldots, x_{n}\right)=\prod_{j=1}^{n-1} r_{1, j}\left(\sum_{l=1}^{j} x_{l}\right) \frac{\bar{K}_{1, j}\left(\sum_{l=1}^{j} x_{l}\right)}{\bar{K}_{1, j+1}\left(\sum_{l=1}^{j} x_{l}\right)} k_{1, n}\left(\sum_{l=1}^{n} x_{l}\right), \quad x_{k} \geq 0, k=1, \ldots, n .
$$

The density function $\ell_{2, n}$ of $\left(X_{2,1}, X_{2,2}, \ldots, X_{2, n}\right)$ is an extension of $(4.5)$ and is given by

$$
\ell_{2, n}\left(x_{1}, \ldots, x_{n}\right)=\prod_{j=1}^{n-1} r_{2, j}\left(\sum_{l=1}^{j} x_{l}\right) \frac{\bar{K}_{2, j}\left(\sum_{l=1}^{j} x_{l}\right)}{\bar{K}_{2, j+1}\left(\sum_{l=1}^{j} x_{l}\right)} k_{2, n}\left(\sum_{l=1}^{n} x_{l}\right), \quad x_{k} \geq 0, k=1, \ldots, n .
$$

In this subsection we derive some extensions and analogs of the results in the previous subsection.

The first result gives conditions under which the epoch times of the two nonhomogeneous birth processes are ordered according to the usual stochastic order. This result may be compared with Theorem 4.2. The following result can be shown to follow from Theorem 2.7 in Shaked and Szekli (1995), however, we provide here a simple direct proof of it. 
Theorem 4.9. Let $X_{i, n}$ be defined as in (4.9) and (4.10). If $Y_{1,1} \leq_{\mathrm{st}} Y_{2,1}$ and if

$$
r_{1, j}(u) \geq r_{2, j}(u+x), \quad u \geq 0, x \geq 0, j \geq 2,
$$

then

$$
\left(X_{1,1}, X_{1,2}, \ldots, X_{1, n}\right) \leq_{\mathrm{st}}\left(X_{2,1}, X_{2,2}, \ldots, X_{2, n}\right), \quad n \geq 1 .
$$

Proof. The result is obvious when $n=1$. So fix an $n \geq 2$. Let $x_{k}^{\prime} \geq x_{k} \geq 0, k=1,2, \ldots, n$. Now, for $j=2, \ldots, n$ we have

$$
\begin{aligned}
& {\left[X_{1, j} \mid X_{1,1}=x_{1}, \ldots, X_{1, j-1}=x_{j-1}\right]==_{\mathbf{s t}}\left[Y_{1, j}-\sum_{k=1}^{j-1} x_{k} \mid Y_{1, j}>\sum_{k=1}^{j-1} x_{k}\right],} \\
& {\left[X_{2, j} \mid X_{2,1}=x_{1}^{\prime}, \ldots, X_{2, j-1}=x_{j-1}^{\prime}\right]=\mathbf{s t}\left[Y_{2, j}-\sum_{k=1}^{j-1} x_{k}^{\prime} \mid Y_{2, j}>\sum_{k=1}^{j-1} x_{k}^{\prime}\right] .}
\end{aligned}
$$

Denote $z=\sum_{k=1}^{j-1} x_{k}$ and $z^{\prime}=\sum_{k=1}^{j-1} x_{k}^{\prime}$. It is not hard to see that (4.13) implies that

$$
\left[Y_{1, j}-z \mid Y_{1, j}>z\right] \leq_{\mathbf{s t}}\left[Y_{2, j}-z^{\prime} \mid Y_{2, j}>z^{\prime}\right] \text { whenever } z^{\prime} \geq z \geq 0, j \geq 2
$$

(in fact, (4.13) and (4.15) are equivalent). Thus the stated result follows from Theorem 4.B.3 in $[19]$.

As a corollary (see a comment following Theorem 4.2) we see that if $Y_{1,1} \leq_{\mathrm{st}} Y_{2,1}$, and if $Y_{1, j} \leq_{\mathrm{hr}} Y_{2, j}, j \geq 2$, and if $Y_{1, j}$ or $Y_{2, j}$ are DFR, $j \geq 2$, then (4.14) holds.

The next result is an extension of Theorem 4.5.

Theorem 4.10. Let $X_{i, n}$ be defined as in (4.9) and (4.10). If $Y_{1, j} \leq_{\mathrm{hr}} Y_{2, j}$, and if $r_{2, j} / r_{1, j}$ is increasing, and if (3.14) holds, and if $r_{1, j}, \frac{\bar{K}_{1, j}}{\bar{K}_{1, j+1}}$ and $k_{1, j}$ or $r_{2, j}, \frac{\bar{K}_{2, j}}{K_{2, j+1}}$ and $k_{2, j}$ are logconvex for all $j \geq 1$, then

$$
\left(X_{1,1}, X_{1,2}, \ldots, X_{1, n}\right) \leq_{\operatorname{lr}}\left(X_{2,1}, X_{2,2}, \ldots, X_{2, n}\right), \quad n \geq 1 .
$$

The proof of Theorem 4.10 is a straightforward extension of the proof of Theorem 4.5, using (4.11) and (4.12) rather than (4.4) and (4.5); we omit the details.

It is worth mentioning that $\frac{\bar{K}_{i, j}}{\bar{K}_{i, j+1}}$ is logconvex if, and only if, $r_{i, j+1}-r_{i, j}$ is increasing.

\section{Some Applications}

In this section we describe some applications of the results of Sections 3 and 4 . The list of applications that we provide below is far from exhaustive, and is given only as an indication of the applicability of the mathematical results. In fact, the results of Sections 3 and 4 provide useful bounds in almost any area where birth processes are used. 


\subsection{Comparisons of generalized Yule birth processes}

A Yule (or a linear) birth process is a birth process with jump intensity from state $n$ to state $n+1$ of the form $(n+1) \lambda$, where $\lambda>0$. Let us consider a generalization $N_{1}=\left\{N_{1}(t), t \geq 0\right\}$ of the Yule process in which the jump intensity at time $t$, given that $n$ jumps have occurred already, is of the form $(n+1) \lambda(t)$. Let $N_{2}=\left\{N_{2}(t), t \geq 0\right\}$ be another generalized Yule process with the corresponding jump intensity $(n+1) \eta(t)$.

The generalized Yule process is a nonhomogeneous birth process. Thus, if the distribution functions $F$ and $G$, that are associated with the failure rate functions $\lambda$ and $\eta$, satisfy $F \leq_{\mathrm{hr}} G$ (that is, $\lambda(u) \geq \eta(u)$ for all $u \geq 0$ ), then, by Theorem 3.9 or 3.10 , it is seen that at any time $t$, there are stochastically at least as many jumps in $N_{1}$ as there are in $N_{2}$ (this is an intuitively clear result that can also be proven directly). If, in addition to $\lambda(u) \geq \eta(u)$ (note that then (3.14) holds too), we also have that $\eta / \lambda$ is increasing, then by Theorem 3.11 the vectors of the first $n$ jumps are ordered in the multivariate likelihood ratio order, and sharper inequalities hold (see, for example, (5.1) below).

If $\lambda$ and $\eta$ satisfy (4.13), that is, $\lambda(u) \geq \eta(u+x)$ for all $x \geq 0$ and $u \geq 0$, then by Theorem 4.9 all the times between births in $N_{1}$ are stochastically smaller than the corresponding times between births in $N_{2}$.

A generalized Yule process may model the spread of a disease, where $n$ is the number of infectives, and $\lambda(t)$ is the rate in which infectives pass the disease to new individuals at time $t$; this rate, in general, depends on the calendar time $t$ - for example, it may change with the seasons of the year (Bailey, 1975). Consider now two generalized Yule processes, with rates $\lambda(t)$ and $\eta(t)$, which model the spread of a disease under two different health measures that are expected to control the spread. The stochastic inequalities described above can direct a health official as to how to fight the spread of a disease, if the official can select between the two measures that control the spread with respective rates $\lambda(t)$ and $\eta(t)$.

A comparison of a generalized Yule process $N_{1}$ (with intensities $r_{1, n}(t)=(n+1) \lambda(t)$ ) with a standard Yule process $N_{2}$ (with intensities $r_{2, n}(t)=(n+1) \eta$, independent of $t$ ) can provide computable upper or lower bounds on various probabilistic quantities of interest that are associated with $N_{1}$. This is based on the fact that the inter-epoch intervals $X_{2,1}, X_{2,2}, \ldots, X_{2, n}, \ldots$ of the standard Yule process $N_{2}$ are independent exponential random variables with rates $\eta, 2 \eta, \ldots, n \eta, \ldots$, and the epoch times $T_{2, n}$ are sums of these independent $X_{2, i}$ 's. For example, suppose that we have under study a generalized Yule process $N_{1}$ as above, and suppose that $\lambda(t)$ is bounded from below by a constant $\eta$ (that is, $\lambda(t) \geq \eta$ for all $t \geq 0$ ). Define $N_{2}$ as the 
standard Yule process with the associated rate $\eta$. Then by Theorems 3.9 or 3.10 , and 4.9 we get $\left(T_{1,1}, \ldots, T_{1, n}\right) \leq_{\mathrm{st}}\left(T_{2,1}, \ldots, T_{2, n}\right)$ and $\left(X_{1,1}, \ldots, X_{1, n}\right) \leq_{\mathrm{st}}\left(X_{2,1}, \ldots, X_{2, n}\right)$, and therefore $E \phi\left(T_{1,1}, \ldots, T_{1, n}\right) \leq E \phi\left(T_{2,1}, \ldots, T_{2, n}\right)$ and $E \phi\left(X_{1,1}, \ldots, X_{1, n}\right) \leq E \phi\left(X_{2,1}, \ldots, X_{2, n}\right)$ for any increasing function $\phi$ for which the expectations exist. For example,

$$
E T_{1, n} \leq E T_{2, n}=\eta^{-1} \sum_{i=1}^{n} i^{-1} .
$$

Another example is provided by Theorem 4.1 of Bunge and Nagaraja (1992). The authors give there an explicit expression for the expected value of the waiting time until the $n$th record occurs when the arrival process is a standard Yule process. If in a particular application the arrival process is a generalized (rather than standard) Yule process, and its associated rate $\lambda(t)$ is bounded from below, then the explicit expression in Theorem 4.1 of Bunge and Nagaraja (1992) provides an upper bound for the expected value of the waiting time until the $n$th record occurs. Another reference in which one can find explicit expressions of probabilistic quantities of interest that are associated with a standard Yule process is the paper by Brown, Ross, and Shorrock (1975). When the rate $\lambda(t)$ that is associated with the generalized Yule process is bounded from below or from above, then these explicit expressions can bound the corresponding probabilistic quantities that are associated with the generalized Yule process. In fact, Brown, Ross, and Shorrock (1975) study a Yule process with immigration (that is, the intensity is of the form $(n+1) \eta+\theta$ ); this process can bound a nonhornogeneous birth process with intensities of the form $r_{n}(t)=(n+1) \lambda(t)+\mu(t)$.

Consider now again the generalized Yule process $N_{1}$ with intensities $r_{1, n}(t)=(n+1) \lambda(t)$, and the standard Yule process $N_{2}$ with intensities $r_{2, n}(t)=(n+1) \eta$, that we described above. If, in addition to $\lambda(t) \geq \eta$, we also have that $\lambda(t)$ is decreasing, then $\eta / \lambda(t)$ is increasing, and by Theorem 3.11 we have $\left(T_{1,1}, \ldots, T_{1, n}\right) \leq_{\operatorname{lr}}\left(T_{2,1}, \ldots, T_{2, n}\right)$. Then, for example, we have

$$
\begin{aligned}
E\left[\phi\left(T_{1,1}, \ldots, T_{1, n}^{-}\right) \mid t_{i}^{0} \leq T_{1, i} \leq t_{i}^{1}, i\right. & =1, \ldots, n] \\
& \leq E\left[\phi\left(T_{2,1}, \ldots, T_{2, n}\right) \mid t_{i}^{0} \leq T_{2, i} \leq t_{i}^{1}, i=1, \ldots, n\right]
\end{aligned}
$$

for all increasing functions $\phi$, whenever $t_{i}^{0}<t_{i}^{1}, i=1, \ldots, n$ (see Theorem 4.E.1 in [19]). Such an inequality does not follow, in general, from the weaker condition $\left(T_{1,1}, \ldots, T_{1, n}\right) \leq_{\mathrm{st}}$ $\left(T_{2,1}, \ldots, T_{2, n}\right)$. See below, in the next paragraph, a possible practical application of (5.1).

When $\lambda(t) \geq \eta$, and $\lambda(t)$ is decreasing, then all the conditions of Theorem 4.10 hold. In order to see it we first note that (3.14) obviously holds because $\lambda(t) \geq \eta$. If $k_{2, j}$ denotes the exponential density with rate $j \eta$ then it is easy to verify that $r_{2, j}, \frac{\bar{K}_{2, j}}{\bar{K}_{2, j+1}}$ and $k_{2, j}$ 
are all logconvex. Thus, from Theorem 4.10 we obtain $\left(X_{1,1}, \ldots, X_{1, n}\right) \leq_{\operatorname{lr}}\left(X_{2,1}, \ldots, X_{2, n}\right)$, where $X_{2,1}, \ldots, X_{2, n}$ are independent exponential random variables as described above. This stochastic inequality is useful in a situation where benefits are derived at any inter-epoch time interval. For example, suppose that the benefit from a realization $\left(x_{1}, \ldots, x_{n}\right)$ of $\left(X_{1,1}, \ldots, X_{1, n}\right)$ is $\phi\left(x_{1}, \ldots, x_{n}\right)$, but the benefits are derived only during an initial period of length $t_{0}$ in any inter-epoch interval. Then the expected benefit from the first $n$ inter-epoch intervals of the generalized Yule process is $E\left[\phi\left(X_{1,1}, \ldots, X_{1, n}\right) \mid X_{1, i} \leq t_{0}, i=1, \ldots, n\right]$, provided the expectation exists. When $\phi$ is increasing then this expectation is bounded from above by $E\left[\phi\left(X_{2,1}, \ldots, X_{2, n}\right) \mid X_{2, i} \leq t_{0}, i=1, \ldots, n\right]$; this follows from $\left(X_{1,1}, \ldots, X_{1, n}\right) \leq_{\text {lr }}$ $\left(X_{2,1}, \ldots, X_{2, n}\right)$ and from Theorem 4.E.1 in [19]. The latter expectation is not hard to compute because $X_{2,1}, \ldots, X_{2, n}$ are independent exponential random variables.

\subsection{Comparisons of load-sharing models}

Consider $n$ items that share a load $L_{1}(t)$ at time $t$. A common model (see Schechner (1984)) is to assume that the failure rate of each item then is $L_{1}(t) / n$. After $i$ items have already failed, each of the remaining $n-i$ items has a load of $L_{1}(t) /(n-i)$. If we denote $r_{1, i}(t)=$ $L_{1}(t) /(n-i+1)$, then it is seen that the failure times $T_{1,1} \leq T_{1,2} \leq \cdots \leq T_{1, n}$ are the epoch times of a nonhomogeneous birth process. Let $L_{2}$ be a second load shared by $n$ similar items.

If $L_{1}(u) \geq L_{2}(u)$ for all $u \geq 0$, then, by Theorem 3.9 or 3.10 , it is seen that at any time $t$, there are stochastically at least as many failures in the first model as there are in the second (this is an intuitively clear result that can also be proven directly). If, in addition to $L_{1}(u) \geq L_{2}(u)$ (note that then (3.14) holds too), we also have that $L_{2} / L_{1}$ is increasing, then by Theorem 3.11 the vectors of the $n$ failure times are ordered in the multivariate likelihood ratio order, and sharper inequalities hold.

If $L_{1}(u) \geq L_{2}(u+x)$ for all $x \geq 0$ and $u \geq 0$, then, by Theorem 4.9, all the times between failures in the first model are stochastically smaller than the corresponding times between failures in the second model.

If the load $L_{2}$ is constant then some probabilistic quantities of interest can be computed explicitly. Thus, when $L_{1}(t)$ is bounded from below or from above, we can use the loadsharing model associated with $L_{2}$ in order to bound some probabilistic quantities of interest involving the model associated with $L_{1}(t)$. For example, Equation (4.9) of Phoenix (1978) gives an explicit expression for the mean of a single member in a load-sharing model with a constant $L_{2}$. If $L_{1}(u) \geq L_{2}$ for all $u \geq 0$ then, using Theorems $3.9,3.10$ and 4.9 , we see 
that (4.9) of Phoenix (1978) provides an upper bound on the corresponding expectation in the model associated with $L_{1}$.

A load-sharing model with a constant $L$ often describes the strength of a bundle of fibers. If the load on the bundle varies with time (for example, the load may be different during the day than during the night) then the general model, in which $L$ depends on $t$, applies.

\subsection{Comparisons of benefits between times of minimal repair}

The repair times of an item that is continuously minimally repaired are the epoch times of a nonhomogeneous Poisson process whose intensity function is the hazard rate function of the lifetime distribution of the item; see, for example, Shaked and Szekli (1995).

Suppose that a manager has to decide which of two items that are continuously minimally repaired is to be used. The selected item (which may be, for example, a computer, a car, or an airplane) can then be used until its next failure. If we denote by $X$ a generic intrafailure interval, then it can be assumed that the benefit derived from the item is an increasing function $\phi(X)$ of the interval (see, for example, a discussion in page 1093 of Shaked and Szekli (1995)). If the choice of the manager is between the $i$ th interval of either of the two items, then Theorems 4.1-4.7 can direct the manager in his choice. For example, if the item is going to be used for the whole duration of the intrafailure interval, then under the conditions of Theorems 4.1 or 4.2 we have $E\left[\phi\left(X_{1, i}\right)\right] \leq E\left[\phi\left(X_{2, i}\right)\right]$, and thus the second item is preferable. If the item can be used only after some fixed burn-in time $x_{0}$, then under the conditions of Theorem 4.4 we have $E\left[\phi\left(X_{1, i}-x_{0}\right) \mid X_{1, i}>x_{0}\right] \leq E\left[\phi\left(X_{2, i}-x_{0}\right) \mid X_{2, i}>x_{0}\right]$, for any fixed $x_{0}$, and thus again the second item is preferable (by (1.B.5) in [19]). Finally, if the item is going to be used only for a fixed subinterval, $\left[x_{0}, x_{1}\right]$ say, of the intrafailure interval, then under the conditions of Theorems 4.5 or 4.7 we have $E\left[\phi\left(X_{1, i}-x_{0}\right) \mid x_{0}<X_{1, i} \leq x_{1}\right] \leq$ $E\left[\phi\left(X_{2, i}-x_{0}\right) \mid x_{0}<X_{2, i} \leq x_{1}\right]$, for any fixed $x_{0}<x_{1}$, and thus the second item is preferable (by (1.C.4) in [19]).

\section{Acknowledgement}

We thank Claude Lefevre and Fabio Spizzichino with whom we had some fruitful conversations which clarified the meaning and the usefulness of the nonhomogeneous birth processes. 


\section{References}

[1] Bagai, I. and Kochar S. C. (1986), On tail-ordering and comparison of failure rates, Communications in Statistics-Theory and Methods 15, 1377-1388.

[2] Bailey, N. T. J. (1975), The Mathematical Theory of Infectious Diseases and Its Applications, Griffin, London.

[3] Bartoszewicz, J. (1985), Dispersive ordering and monotone failure rate distributions, Advances in Applied Probability 17, 472-474.

[4] Baxter, L. A. (1982), Reliability applications of the relevation transform, Naval Research Logistics Quarterly 29, 323-330.

[5] Brown, M., Ross, S. and Shorrock, R. (1975), Evacuation of a Yule process with immigration, Journal of Applied Probability 12, 807-811.

[6] Bunge, J. A. and Nagaraja, H. N. (1992), Exact distribution theory for some point process record models, Advances in Applied Probability 24, 20-44.

[7] Gupta, R. C. and Kirmani, S. N. U. A. (1988), Closure and monotonicity properties of nonhomogeneous Poisson processes and record values, Probability in the Engineering and Informational Sciences 2, 475-484.

[8] Kamps, O. (1995), A Concept of Generalized Order Statistics, B. G. Taubner, Stuttgart.

[9] Karlin, S. (1968), Total Positivity, Stanford University Press, Palo Alto, CA.

[10] Kochar, S. C. (1996a), Some results on interarrival times of nonhomogeneous Poisson processes, Probability in the Engineering and Informational Sciences 10, 75-85.

[11] Kochar, S. C. (1996b), A note on dispersive ordering of record values, Calcutta Statistical Association Bulletin 46, 63-67.

[12] Pellerey, F., Shaked, M. and Zinn, J. (1999), Nonhomogeneous Poisson processes and logconcavity, Technical Report, Department of Mathematics, University of Arizona, Tucson.

[13] Phoenix, S. L. (1978), The asymptotic time to failure of a mechanical system of parallel members, SIAM Journal of Applied Mathematics 34, 227-246. 
[14] Rowell, G. and Siegrist, K. (1998), Relative aging of distributions, Probability in the Engineering and Informational Sciences 12, 469-478.

[15] Schechner, Z. (1984), A load-sharing model: the linear breakdown rule, Naval Research Logistics Quarterly 31, 137-144.

[16] Sengupta, D. and Deshpande, J. V. (1994), Some results on the relative ageing of two life distributions, Journal of Applied Probability 31, 991-1003.

[17] Shaked, M. and Shanthikumar, J. G. (1991), Dynamic multivariate mean residual life functions, Journal of Applied Probability 28, 613-629.

[18] Shaked, M. and Shanthikumar, J. G. (1993), Dynamic conditional marginal distributions in reliability theory, Journal of Applied Probability 30, 421-428.

[19] Shaked, M. and Shanthikumar, J. G. (1994), Stochastic Orders and Their Applications, Academic Press, San Diego.

[20] Shaked, M. and Szekli, R. (1995), Comparison of replacement policies via point processes, Advances in Applied Probability 27, 1079-1103.

[21] Shanthikumar, J. G. and Yao, D. D. (1986), The preservation of the likelihood ratio ordering under convolution, Stochastic Processes and Their Applications 23, 259-267. 